\title{
Absorption and related optical dispersion effects on the spectral response of a surface plasmon resonance sensor
}

\author{
Mohamed Nakkach, ${ }^{1,2, \star}$ Pierre Lecaruyer, ${ }^{1}$ Fabrice Bardin, ${ }^{1}$ Jaouhar Sakly, ${ }^{3}$ \\ Zohra Ben Lakhdar, ${ }^{2}$ and Michael Canva ${ }^{1}$ \\ ${ }^{1}$ Laboratoire Charles Fabry de I'Institut d'Optique, Institut d'Optique Graduate School, Univ. Paris-Sud, \\ CNRS, Campus Polytechnique, RD 128, 91127 Palaiseau cedex, France \\ ${ }^{2}$ Laboratoire de Spectroscopie Atomique Moléculaire et Applications, Fac. Sc. Tunis 1060, Tunisie \\ ${ }^{3}$ Institut National des Sciences Appliquées et de Technologie, Centre urbain nord, B.P. 676 Cedex Tunis, Tunisie \\ ${ }^{*}$ Corresponding author: mohamed.nakkach@u-psud.fr
}

Received 15 July 2008; accepted 15 August 2008;

posted 20 October 2008 (Doc. ID 98896); published 13 November 2008

\begin{abstract}
Surface plasmon resonance (SPR) sensing is an optical technique that allows real time detection of small changes in the physical properties, in particular in the refractive index, of a dielectric medium near a metal film surface. One way to increase the SPR signal shift is then to incorporate a substance possessing a strong dispersive refractive index in the range of the plasmon resonance band. In this paper, we investigate the impact of materials possessing a strong dispersive index integrated to the dielectric medium on the SPR reflectivity profile. We present theoretical results based on chromophore absorption spectra and on their associated refractive index obtained from the Lorentz approach and KramersKrönig equations. As predicted by the theory, the experimental results show an enhancement of the SPR response, maximized when the chromophore absorption band coincides with the plasmon resonant wavelength. This shows that chromophores labeling can provide a potential way for SPR response enhancement. (C) 2008 Optical Society of America
\end{abstract}

OCIS codes: $\quad 120.0120,160.4760,240.6680,310.6188,310.6870$.

\section{Introduction}

Surface plasmon resonance (SPR) technique has become widely used today to detect and to characterize in real time and without labels biomolecular interactions such as DNA-DNA [1-3] and protein-protein [4-6]. SPR biosensors are able to detect small changes in the refractive index or in the thickness of thin layers induced by a variation of concentration or by interaction between biomolecules occurring very near the sensitive surface. The Kretschmann configuration [7] is the most used to generate surface plasmon waves based on two principal interrogation techniques: angular and spectral interrogations [8].

0003-6935/08/336177-06\$15.00/0

(C) 2008 Optical Society of America
In general, the methods that are used to characterize dielectric media are chosen under the assumption that the refractive index remains constant in the visible range, meaning that potential dispersion is not taken into account.

In the last few years, the SPR technique has also been used to study the absorption spectrum of thin layers near a metal film. In this case, the dielectric medium has a refractive index variation around its absorption band. The angular interrogation technique has been used to study the effect of dye doped polymer particles [9] or to investigate the sensing of ion-selective membranes [10]. These previous studies have shown an increase in the minimum of reflectivity as a function of the dye concentration, as well as a resonance angular shift. This shift is due to variations in the resonance conditions depending 
on the incident wavelength [10]. Spectral interrogation has been used to investigate the absorption spectroscopy of molecular absorbates [11] and of absorbing liquids [12]. This technique has also been used to study the enhancement of the SPR sensitivity using refractive index dispersion of a dye solution [13] and coupling between surface plasmon and exciton in organic semiconductors [14]. A significant change in the shape of the resonance curve [13] and a modification of the minimal reflectivity [12] have been directly observed around the absorption wavelength. The spectral interrogation technique allows direct measurements of the spectral attenuation and of the plasmon profile modifications.

In this paper, we present the effect of chromophores bounded to DNA target molecules on the SPR biosensor performance. In particular, we show theoretically and experimentally that the chromophores presenting an absorption band in the spectral absorption of the plasmon induce strong refractive index changes which can be clearly observable by the SPR system response.

\section{Simulation of Spectral Response}

\section{A. Introduction to Surface Plasmon Resonance}

Surface plasmon resonance technique is a surface sensitive optical method, based upon evanescent wave sensing techniques, wherein a light source is directed via a coupling prism or grating toward a thin metal film exhibiting conduction band electrons that can be approximated as a free electron plasma. Under certain resonance conditions, which are very dependent on the local environment, the electron plasma absorbs the energy of the excitation source, creating oscillations known as surface plasmon waves at the metal surface $[15,16]$. This absorption is revealed through a sharp decrease in the reflected light amplitude and it depends on the incident angle, wavelength, and refractive index around the metal/ dielectric interface.

The high sensitivity of SPR biosensors is provided by the high dependence of the coupling coefficients on small refractive index changes induced by biomolecular interactions at the metal/dielectric interface. Applied to biochips, this means that each biomolecule interacting within the vicinity of the metallic layer induces an increase in the density of molecules at the interface, leading to a change in refractive index.

\section{B. Plasmon Theory}

In the Kretschmann configuration, the energy of the illuminating beam is coupled at the interface by means of a glass prism. Given a TM-polarized collimated beam incident on the structure at a given angle of incidence $(\theta)$ and a wavelength $(\lambda)$ that ensures phase matching between the parallel component of the wave vector of the incident beam and the wave vector of the surface plasmon wave, the energy can be transferred from the light beam to the plasmon mode. Because the plasmon is necessarily TM polarized, no such transfer occurs for the TE mode.

SPR existence is driven by equations characterizing the excitation conditions of the plasmon wave. First, it requires a total internal reflection without any metal layer, which means, considering Snell's law, that

$$
n_{\text {prism }} \sin \theta_{\text {prism/dielectric }}>n_{\text {dielectric }} \text {. }
$$

Moreover, the plasmon wave propagating at the surface is characterized by a plasmon wave vector $K_{\mathrm{SPR}}$ calculated as

$$
K_{\mathrm{SPR}}(\omega)=\operatorname{Re}\left(\sqrt{\frac{\varepsilon_{m}(\omega) \varepsilon_{d}(\omega)}{\varepsilon_{m}(\omega)+\varepsilon_{d}(\omega)}}\right) \frac{\omega}{c},
$$

where $\omega$ is the frequency of the light, $c$ is the speed of light in a vacuum, and $\varepsilon_{m}$ and $\varepsilon_{d}$ are the permittivities of the metal and dielectric, respectively.

The longitudinal projection of the excitation wave vector $K_{x}$ can be written as

$$
K_{x}=\frac{\omega}{c} n_{\text {prism }}(\omega) \sin \theta
$$

where $\theta$ is the incidence angle and $n_{\text {prism }}$ is the refractive index of the prism at frequency $\omega$.

Finally, the incident light is coupled to the plasmon when its wave vector $K_{x}$ is equal to $K_{\mathrm{SPR}}$. It leads to an energy transfer from the incident light to plasmon modes. Equations (2) and (3) illustrate the dependence of the SPR signal on the excitation wavelength and on the incident angle. In addition to these two parameters $(\theta, \omega)$, the signal depends on the twospace dimensions $(x, y)$, time $(t)$, and on the light polarization. This multidimensional aspect of the new generation of SPR sensors was presented in previous works [17].

\section{Calculation with the Rouard Method}

To model the SPR reflectivity of a thin film stack, numerical simulations have been implemented using the extended Rouard approach adapted to nontransparent media, detailed in a recent publication [18]. The main advantage of this method is its simple implementation and generalization to as many layers as necessary (dielectric media or metals) by means of recursive loops and reflectance Fresnel coefficients at the interfaces. It was used to optimize multilayer film parameters in view of improving the sensitivity of SPR biosensors [19].

In this work, the multilayer stack is composed by the base of a SF11 glass prism, a $1 \mathrm{~nm}$ thick chromium layer, a $58 \mathrm{~nm}$ thick gold film, and a semiinfinite water medium. The spectral refractive index of the SF11 prism and of the gold film were respectively calculated with Sellmeier's equations [20] and Corn's data [21]. The refractive index of the chromium was taken from the SOPRA database [22] and the refractive index of water was estimated to be 1.33 in the visible range. 


\section{Simulation of Absorption Band Effects}

The SPR wavelength shift depends on the refractive index shift of the dielectric medium in contact with the metal layer. Therefore, one way to increase the SPR signal shift is to incorporate a substance possessing a high refractive index. The use of a dye solution presenting a strong dispersion has been demonstrated to be an efficient way to enhance the sensitivity of a SPR sensor [13]. Labeling of a DNA target molecule with a chromophore presenting strong dispersion, and therefore strong refractive index variations, in the range of the SPR resonance would induce an increase in the signal shift.

The model developed for the determination of the SPR reflectivity variation as a function of the adsorbed thin layer thickness [18] has been extended to take into account the spectral dispersion of the adsorbed layer refractive index. The functionalization and the hybridization processes are summarized in Fig. 1. Simplified steps have been used in the model. Figure 2(a) shows the initial plasmon spectrum localized at $654 \mathrm{~nm}$ before the functionalization process (the coupling angle was $36^{\circ} 24^{\prime}$ ). After the functionalization of the DNA probe, the plasmon spectrum shifts slightly to the higher wavelength, inducing a reflectivity variation shown in Fig. 2(b). The probe is modeled as a biomolecular dielectric film at the interface between the prism/chromium/gold multilayer and the semi-infinite dielectric (buffer solution) and is considered as a layer having a refractive index of 1.46 [23] and a thickness of $1.05 \mathrm{~nm}$ as extracted from the reflectivity variation spectrum presented in Fig. 2(b). According to [19], the refractive indices of the single and double strand DNA are 1.46 and 1.53 , respectively. The hybridization of the label-free target molecules ( 25 bases) to the probe ( 35 bases) is taken into account by considering a refractive index change on the target length to 1.53 . The refractive index on the 10 nonhybridized other bases was kept to 1.46. To fit the experimental hybridization mea-

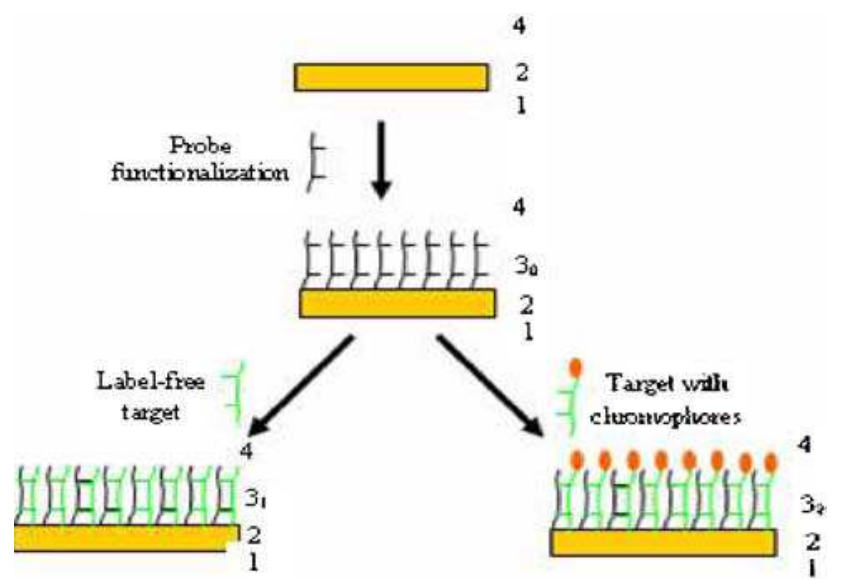

Fig. 1. (Color online) Schematic representation of the probe functionalization and of the biomolecule hybridization with and without labels. The different layers are represented by 1 , prism; 2 , gold; $3_{0}, \quad$ dielectric $=$ probe layer; $3_{1}, \quad 3_{0}+$ target; $3_{2}, \quad 3_{0}+$ target + chromophore; and 4, semi-infinite buffer solution.
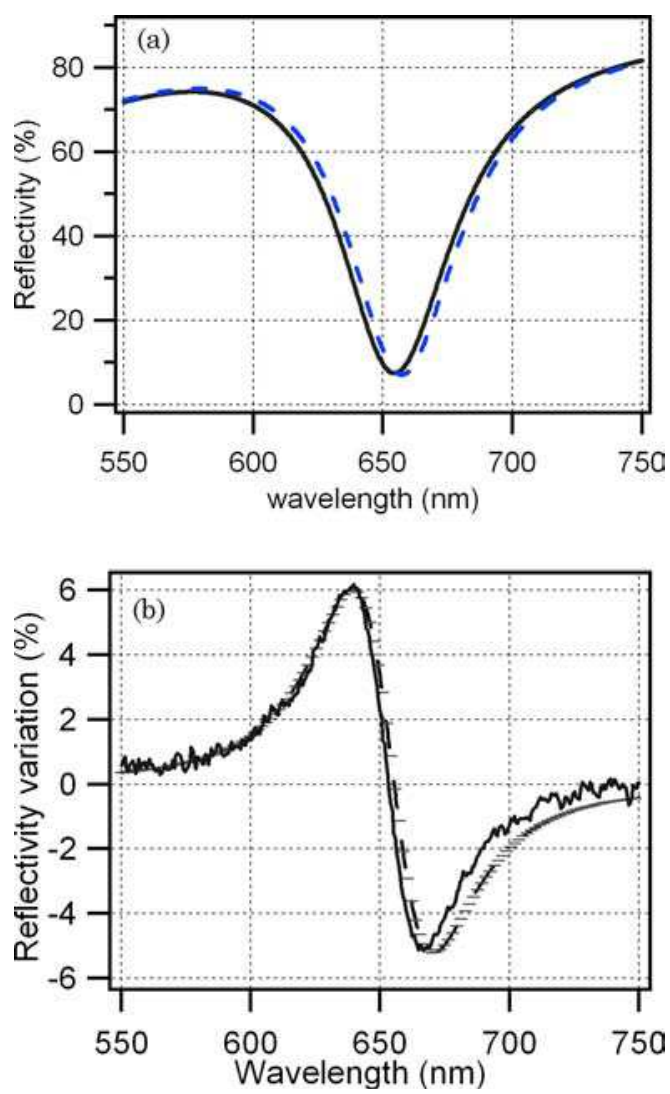

Fig. 2. (Color online) (a) Plasmon spectrum before (solid curve) and after (dashed curve) the functionalization of the probe. (b) Reflectivity variation after the functionalization of the probe molecule on the surface. The solid and the dashed lines are the experimental and the theoretical responses, respectively.

surement with the theoretical results, the hybridization rate was estimated to be $25 \%$, leading to a thickness of $1.21 \mathrm{~nm}$ and a global refractive index of 1.47. The resonance wavelength of the configuration with label-free target is around $657 \mathrm{~nm}$. The integration of the chromophore to the target has then been taken into account by varying the target refractive index around 1.47, as according to the refractive index dispersion calculated by Eq. (4).

The incorporation effect of four commonly used chromophores, namely Rhodamine 6G (Rh), Cy3, Texas Red (TR), and Cy5 has been investigated. These chromophores present two absorption bands [24]. Their associated resonance wavelength $\left(\lambda_{\max }\right)$ and their full width at half-maximum $\left(\gamma_{1}\right)$ are given in Table 1 . The Lorentz equation approach has been used to approximate the complex refractive index $\left(\varepsilon_{d}\right)$ for the used chromophores as detailed in [25], by incorporating the two absorption bands:

$$
\begin{aligned}
\varepsilon_{d}= & \varepsilon_{\text {bulk }} \\
& +\frac{1}{\alpha_{d}}\left[\frac{\omega_{p}^{2}}{\alpha_{1}} \frac{1}{\left(\omega_{1}^{2}-\omega^{2}-i \omega \gamma_{1}\right)}\right. \\
& \left.+\frac{\omega_{p}^{2}}{\alpha_{2}} \frac{1}{\left(\omega_{2}^{2}-\omega^{2}-i \omega \gamma_{2}\right)}\right],
\end{aligned}
$$


Table 1. Chromophore Spectral Absorption Parameters used in the Simulation

\begin{tabular}{|c|c|c|c|c|c|c|c|c|}
\hline \multirow[b]{2}{*}{ Chromophore } & \multicolumn{7}{|c|}{ Simulation Parameters } & \multirow{2}{*}{$\frac{\text { Literature Data [26] }}{\varepsilon}$} \\
\hline & $\begin{array}{c}\lambda_{1 \max } \\
(\mathrm{nm})\end{array}$ & $\begin{array}{c}\gamma_{1} \\
(\mathrm{~nm})\end{array}$ & $\begin{array}{l}\lambda_{2 \max } \\
(\mathrm{nm})\end{array}$ & $\begin{array}{c}\gamma_{2} \\
(\mathrm{~nm})\end{array}$ & $\alpha_{d}$ & $\alpha_{1}$ & $\alpha_{2}$ & \\
\hline Rhodamine $6 \mathrm{G}$ & 500 & 75 & 525 & 25 & 15 & 12 & 13 & 116000 \\
\hline Cy3 & 520 & 40 & 548 & 20 & 10 & 20 & 30 & 150000 \\
\hline Texas Red & 560 & 60 & 595 & 20 & 18 & 19 & 11 & 84000 \\
\hline Cy5 & 610 & 84 & 650 & 28 & 17 & 6 & 4 & 250000 \\
\hline
\end{tabular}

where $\omega_{1}$ and $\omega_{2}$ are the resonance frequencies at $\lambda_{1 \max }$ and $\lambda_{2 \max }$ of the two absorption bands, respectively, $\omega_{p}$ is the plasma frequency, $\omega$ is the frequency of observation, and $\varepsilon_{\text {bulk }}$ is the bulk dielectric coefficient. Compared to Eq. 13 of [21], a dilution coefficient $\alpha_{d}$ calculated from the ratio of the chromophore molecular weight and from the total molecular weight of the labeled target (presented in Table 2) was added. We also introduced two coefficients $-\bar{\alpha}_{1}$ and $\alpha_{2}$, presented in Table 1 -to adjust the absorption of the two bands of each chromophore relative to the extinction coefficient given in the literature. These coefficients also take into account the environmental influence on absorption in this case when the labeled target is hybridized on the chip surface.

Figure 3 presents the theoretical calculation of the optical absorption and dispersion characteristics of the chromophores and the associated SPR responses while incorporating these chromophores into the adsorbed thin layer. These calculations take into account both real and imaginary parts of their associated refractive index.

Figure 3(a) shows the imaginary part of the refractive index (which is directly related to the absorption spectrum) of the four dyes attached to the target molecules. Figure 3(c) shows the dispersive refractive index around $1 . \overline{47}$ of the four chromophores calculated with the Lorentz equation. The strong refractive index changes due to the chromophores induce variations in the SPR reflectivity spectra mainly in the wavelength range of the intersection between the absorption spectra and the resonance spectra as shown in Fig. 3(c). Figure 3(d) illustrates the reflectivity variation calculated from the SPR spectra before and after the interaction. The $\mathrm{Cy} 3, \mathrm{Rh}$, and TR chromophores have therefore no significant influence on the plasmon resonance curve since their dispersion spectrum is out of the spectral range of the plasmon resonance wavelength. However, since the Cy5 chromophore presents a strong refractive index change in the spectral range of the plasmon resonance spectrum $(657 \mathrm{~nm})$, a clear enhancement of the SPR signal is observed around $651 \mathrm{~nm}$.

\section{Experimental Validation of Spectral Effect}

We carried out a series of experiments aimed at observing the effect of chromophore absorption when a dye is incorporated onto target molecules. We functionalized probes presenting the M470V wild type formed by the following sequence: biotin $5^{\prime}(\mathrm{T}) 10$ TTCTAATGATGATTA3' [1] onto the gold surface. Each chromophore was attached to a target complementary to the M470V-WT (wild type) probe.

A schematic diagram of the SPR apparatus is shown in Fig. 4. A collimated, polychromatic light source passes through a polarizer and illuminates the functionalized gold surface in contact with a hybridization cell at a controlled incident angle. The reflected light is then coupled into a spectrum analyzer instrument via an optical fiber. The full spectral resonance profile is then measured in real time. This configuration allows the dynamic measurement of the full spectral resonance profile on one channel (spectral monosensor configuration).

Figure 5(a) shows SPR spectra variations after binding of targets without labels, with Cy3, TR, $\mathrm{Rh}$, and with Cy5 molecules measured at a fixed incident angle on the prism. As predicted by the simulation presented in Section 2, we clearly observe an enhancement of the response by about $160 \%$ only when using the target attached with Cy5 chromophore. This chromophore presents an absorption band in the spectral range of the SPR spectrum, whereas the other chromophore dispersions are out of the spectral range of the plasmon resonance wavelength. Therefore, no significant effect is observed on the signal. At a fixed wavelength, the reaction kinetics presented in Fig. 5(b) show: (i) an amplification response of the target attached to Cy5 due to its maximum refractive index dispersion at

Table 2. Molecular Weight of the Probe and Targets Involved in the Interaction

\begin{tabular}{|c|c|c|c|c|c|c|}
\hline & \multirow{2}{*}{$\begin{array}{c}\text { DNA Probe } \\
(35 \text { bases })\end{array}$} & \multicolumn{5}{|c|}{$\begin{array}{c}\text { DNA Target } \\
\text { (25 bases })\end{array}$} \\
\hline & & Unlabeled & $\begin{array}{l}\text { Labeled with } \\
\text { Rhodamine } 6 \mathrm{G}\end{array}$ & $\begin{array}{l}\text { Labeled with } \\
\text { Texas Red }\end{array}$ & $\begin{array}{c}\text { Labeled with } \\
\text { Cy3 }\end{array}$ & $\begin{array}{l}\text { Labeled with } \\
\text { Cy5 }\end{array}$ \\
\hline Molecular weight (g/mol) & 11747 & 7671 & 8230 & 8491 & 8117 & 8143 \\
\hline
\end{tabular}



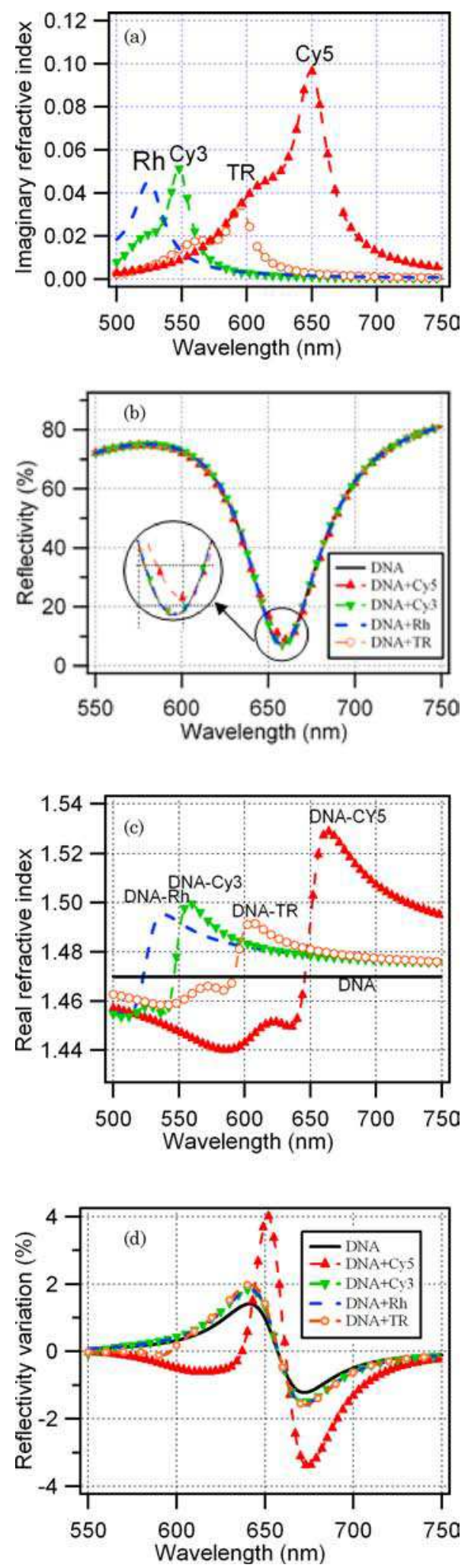

Fig. 3. (Color online) Dispersion spectra of the Rh, Cy3, TR, and Cy5 chromophores: (a) absorption spectra and (b) SPR reflectivity after interaction with target molecules without label, with $\mathrm{Rh}$, Cy3, TR, and Cy5 in a SF11/chromium/gold/dielectric/water Kretschmann configuration. (c) Calculated variation of the refractive index. (d) Differential SPR reflectivity obtained by subtracting reflectivity curves before and after interaction. The SPR curves have been calculated for an external incident angle of $36^{\circ} 24^{\prime}$ on the prism.

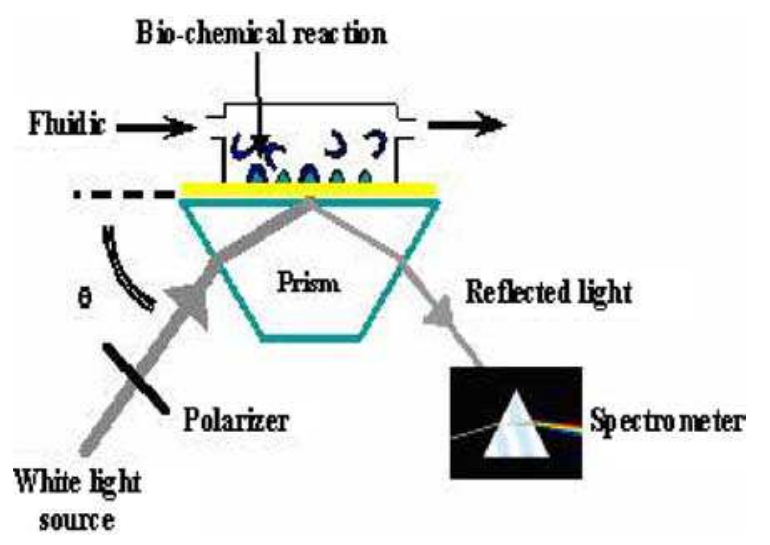

Fig. 4. (Color online) Experimental setup of the spectral mono channel sensor.

$652 \mathrm{~nm}$, (ii) a smaller amplification induced by the other chromophores compared to the label-free target due to the small difference in their refractive index at $643 \mathrm{~nm}$.

\section{Conclusion}

Target absorption effect on the spectral response of a SPR sensor has been highlighted. We demonstrated,
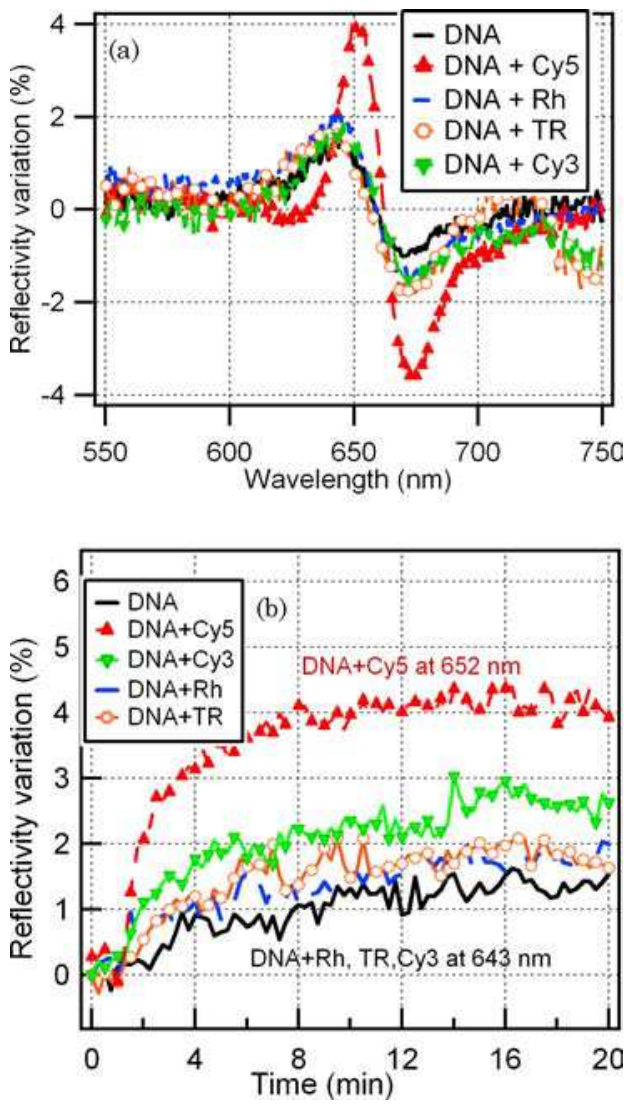

Fig. 5. (Color online) (a) Reflectivity variation spectra after interaction with DNA target, without label, with Cy3, TR, Rh, and with Cy5. The reflectivity spectra are compared with the spectrum before interaction. (b) Reaction kinetic at maximum reflectivity variation at $652 \mathrm{~nm}$ of the target labeled with Cy5 and at $642 \mathrm{~nm}$ of target without label, labeled with $\mathrm{Cy} 3$, TR, and $\mathrm{Rh}$. 
both theoretically and experimentally, that labeling DNA target molecules with chromophore induces significant spectral SPR response changes. This enhancement $(160 \%)$ is clearly due to their intrinsic strong spectral refractive index variation and not to the chromophore weight added to the target because the chromophore represents only $5.8 \%$ of the target weight. We have also shown that this phenomenon appears only when the chromophore presents an absorption band in the spectral range of the plasmon resonance coupling spectrum. The use of specific chromophores provides a potential way for SPR response enhancement, and these initial results suggest that this phenomenon can also be used in SPR imaging, in particular on SPR dynamic biochips. Finally, the influence of labels on the kinetics or on the equilibrium of biomolecular binding events has to be clarified to take full advantage of the enhanced sensitivity induced by dispersion from a chromophore. In addition, these results suggest a possible identification and selectivity tool for labeled targets with chromophores by tuning the resonance conditions. It also opens the way to SPR spectroscopy.

The authors acknowledge French public funding support through the ANR (Agence Nationale de la Recherche) PNANO Program.

\section{References}

1. I. Mannelli, V. Courtois, P. Lecaruyer, G. Roger, M. C. Millot, M. Goosens, and M. Canva, "Surface plasmon resonance imaging (SPRI) system and real-time monitoring of DNA biochip for human genetic mutation diagnosis of DNA ampllified samples," Sens. Actuators B 119, 583-591 (2006).

2. J. Spadavecchia, M. G. Manera, F. Quaranta, P. Siciliano, and R. Rella, "Surface plasmon resonance imaging of DNA based biosensors for potential applications in food analysis," Biosens. Bioelectron. 21, 894-900 (2005).

3. M. G. Manera, J. Spadavecchia, A. Leone, F. Quaranta, R. Rella, D. Dell'atti, M. Minunni, M. Mascini, and P. Siciliano, "Surface plasmon resonance imaging technique for nucleic acid detection," Sens. Actuators B 130, 82-87 (2008).

4. M. Kim, K. Park., E. J. Jeong, Y. B. Shin, and B. H. Chung, "Surface plasmon resonance imaging analysis of proteinprotein interactions using on-chip-expressed capture protein," Anal. Biochem. 351, 298-304 (2006).

5. T. Mori, K. Inamori, Y. Inoue, X. Han, G. Yamanouchi, T. Niidome, and Y. Katayama, "Evaluation of protein kinase activities of cell lysates using peptide microarrays based on surface plasmon resonance imaging," Anal. Biochem. 375, 223-231 (2008).

6. J. S. Yuk, H. S. Kim, J. W. Jung, S. H. Jung, S. J. Lee, W. J. Kimb, J. Han, Y. M. Kim, and K. S. Ha, "Analysis of protein interactions on protein arrays by a novel spectral surface plasmon resonance imaging," Biosens. Bioelectron. 21, 1521-1528 (2006).
7. E. Kretschmann and H. Raether, "Radiative decay of nonradiative surface plasmons excited by light," Z. Naturforsch 23, 2135-2136 (1968).

8. J. Homola, "Surface plasmon resonance sensors for detection of chemical and biological species," Chem. Rev. 108, 462-493 (2008).

9. H. Komatsu, M. Miyachi, E. Fujii, D. Citterio, K. Yamada, Y. Sato, K. Kurihara, H. Kawaguchi, and K. Suzuki, "SPR sensor signal amplification based on dye-doped polymer particles," Sci. Tech. Adv. Mater. 7, 150-155 (2006).

10. K. Kurihara, K. Nakamura, E. Hirayama, and K. Suzuki, "An absorption-based surface plasmon resonance sensor applied to sodium ion sensing based on an ion-selective optode membrane," Anal. Chem. 74, 6323-6333 (2002).

11. S. Wang, S. Boussad, and N. J. Tao, "Surface plasmon resonance enhanced optical absorption spectroscopy for studying molecular adsorbates," Rev. Sci. Instrum. 72, 3055-3060 (2001).

12. A. A. Kolomenskii, P. D. Gershon, and A. Schuessler, "Surfaceplasmon resonance spectrometry and characterization of absorbing liquids," Appl. Opt. 39, 3314-3320 (2000).

13. A. Hanning, J. Roeraade, J. J. Delrow, and R. C. Jorgenson, "Enhanced sensitivity of wavelength modulated surface plasmon resonance devices using dispersion from a dye solution," Sens. Actuators B 54, 25-36 (1999).

14. C. Bonnand, J. Bellesa, and J. C. Plénet, "Study of strong coupling between surface plasmon and exciton in an organic semiconductor," J. Non-Cryst. Solids 352, 1683-1685 (2006).

15. U. Fano, "The theory of anomalous diffraction gratings and of quasi-stationary waves on metallic surfaces (Sommerfeld's waves)," J. Opt. Soc. Am. 31, 213-222 (1941).

16. A. Otto, "Excitation of surface plasma waves in silver by the method of frustrated total reflection," Z. Phys. 216, 398-410 (1968).

17. P. Lecaruyer, I. Mannelli, V. Courtois, M. Goossens, and M. Canva, "Surface plasmon resonance imaging as a multidimensional surface characterization instrument: application to biochip genotyping," Anal. Chim. Acta 573-574, 333-340 (2006).

18. P. Lecaruyer, E. Maillart, M. Canva, and J. Rolland, "Generalization of the Rouard method to an absorbing thin-film stack and application to surface plasmon resonance," Appl. Opt. 45, 8419-8423 (2006).

19. P. Lecaruyer, M. Canva, and J. Rolland, "Metallic film optimization in a surface plasmon resonance biosensor by the extended Rouard method," Appl. Opt. 46, 2361-2369 (2007).

20. http://www.tsukasa-co.jp/product/optics/documents/ CVI_Appendix.pdf.

21. http://unicorn.ps.uci.edu/calculations/fresnel/audata.txt.

22. http://www.sopra-sa.com/index2.php?goto=d1\&rub=4.

23. S. Elhadj, G. Singh, and R. F. Saraf, "Optical properties of immobilized DNA monolayer from 255 to $700 \mathrm{~nm}$," Langmuir 20, 5539-5543 (2004).

24. http://www.eurogentec.com/EGT/files/Oligonucleotides.pdf.

25. P. Prêtre, L.-M. Wu, A. Knoesen, and J. D Swalen, "Optical properties of nonlinear polymers: a method for calculation," J. Opt. Soc. Am. 15, 359-368 (1998).

26. home.earthlink.net/ fluorescentdyes/ McNamara2007FluorophoresTable.xls. 